\title{
Population Structure and Diversity in Valencia Peanut Germplasm Collection
}

\author{
Pratibha Kottapalli, Hari D. Upadhyaya, Kameswara Rao Kottapalli, Paxton Payton, Sangam Dwivedi, \\ Mark Burow, Kalule Okello David, Soum Sanogo, and Naveen Puppala
}

\begin{abstract}
Valencia peanuts [Arachis hypogaea L. subsp. fastigiata Waldron var. fastigiata (Waldron) Krapov. \& W. C. Greg.] are well known for their in-shell market value. Assessment of genetic diversity is key to the success of developing improved cultivars with desirable agronomic and quality traits. Seventy-eight U.S. Valencia core collection accessions together with 36 Valencia accessions representing the global peanut mini-core collection were used to study population structure and diversity and to identify genetically diverse Valencia germplasm for use in peanut breeding. Fifty-two simple sequence repeats loci amplified 683 alleles, with an average of 13 alleles per locus. The mean polymorphism information content and gene diversity, respectively, were 0.270 and 0.335 . The pairwise genetic distance ranged from 0.143 to 0.474 , with an average of 0.631 . Neighbor-joining clustering, principal coordinate analysis, and STRUCTURE analysis consistently separated the Valencia germplasm into five clusters with two distinct major groups. The first major group consisted of genotypes from South America (64\%) with few accessions from Africa, North America, Caribbean, and European regions. The second group consisted of accessions mostly from diverse regions of Africa, North and South America, Asia, and the Caribbean. However, the structuring was not related to the geographic origin and several admixtures were observed. The information generated in this study and phenotyping of this material for biotic and abiotic stress responses and yield-quality traits will facilitate selection of trait-specific, genetically diverse parents for developing Valencia peanut cultivars with a broad genetic base.
\end{abstract}

P. Kottapalli and N. Puppala, New Mexico State Univ., Agricultural Science Center at Clovis, Clovis, NM 88101; H.D. Upadhyaya and S. Dwivedi, International Crops Research Institute for Semi-Arid Tropics (ICRISAT), Patancheru, PO 502324, Andhra Pradesh, India; K.R. Kottapalli and P. Payton, USDA-ARS Cropping Systems Research Lab., Lubbock, TX 79415; K.R. Kottapalli and M. Burow, Texas Tech Univ., Dep. of Plant and Soil Science, Lubbock, TX 79409; M. Burow, Texas AgriLife Research, Texas A\&M System, Lubbock, TX 79403; K.O. David, National Semi-Arid Resources Research Institute, P.O. Box Soroti, Serere, Uganda; S. Sanogo, Dep. of Entomology, Plant Pathology, and Weed Science, New Mexico State Univ., Las Cruces, NM 88003. Received 3 Aug. 2010. ^Corresponding author (npuppala@nmsu.edu).

Abbreviations: NJ, neighbor joining; PCoA, principal coordinate analysis; PCR, polymerase chain reaction; PIC, polymorphism information content; SSR, simple sequence repeats.

C Ultivated peanut (Arachis hypogaea L.), also known as groundnut, is an important food and oil-producing legume, grown annually on 23.51 million ha with a production of $35.5 \mathrm{Mt}$ (FAOSTAT, 2008) in tropical and subtropical regions of the world (mainly in the Americas, Asia, and Africa). China, India, Nigeria, and the United States are the major peanut producers, accounting for $68.7 \%$ of global production (FAOSTAT, 2008). Peanut ranks sixth among the major oil-producing crops after soybean [Glycine $\max (\mathrm{L}$.$) Merr.], oil palm (Elaeis guineensis Jacq.), cotton (Gossypium$ hirsutum L.), coconut (Cocos nucifera L.), and rapeseed (Brassica napus L.), and has the potential to be used as a biodiesel crop. Peanut is rich in oil, protein, minerals, and vitamins, making it a valued crop for direct human consumption, while its fodder is highly nutritious and palatable animal feed. Peanut production in the United States is primarily targeted to the confectionary industry and for production

Published in Crop Sci. 51:1089-1100 (2011).

doi: 10.2135/cropsci2010.08.0452

Published online 29 Mar. 2011.

(C) Crop Science Society of America | 5585 Guilford Rd., Madison, WI 53711 USA

All rights reserved. No part of this periodical may be reproduced or transmitted in any form or by any means, electronic or mechanical, including photocopying, recording, or any information storage and retrieval system, without permission in writing from the publisher. Permission for printing and for reprinting the material contained herein has been obtained by the publisher. 
of peanut butter. The four market types (i.e., Virginia, Runner, Spanish, and Valencia) grown in the United States are characterized by distinct growth habit, pod/seed size, and flavor attributes (Isleib et al., 2001). Valencia [Arachis hypogaea L. subsp. fastigiata Waldron var. fastigiata (Waldron) Krapov. \& W. C. Greg.] peanuts, which constitute only $1-2 \%$ of the total U.S. market and are almost exclusively cultivated in New Mexico and Texas, have high in-shell market value, possess sweet flavor as desired by the confectionary industry, and remain a viable niche crop for production in these regions.

Cultivated peanut $(2 n=4 x=40)$ is an allotetraploid $(\mathrm{AABB})$, native to South America, whose origin is attributed to a single hybridization event involving diploid wild species Arachis duranensis Krapov. \& W. C. Greg. (AA-genome) and $A$. ipaensis Krapov. \& W. C. Greg. (BB-genome) followed by a spontaneous chromosome duplication (Kochert et al., 1996; Hopkins et al., 1999; Fávero et al., 2006; Seijo et al., 2007). Cultivated peanut has six botanical varieties, each differentiated by the presence or absence of flowers on the main axis and unique pod and seed characteristics (Gregory et al., 1980; Krapovickas and Gregory, 1994). Although cultivated peanut has considerable variability in morphological traits, several studies reported a low level of molecular diversity (Baichi-Hall et al., 1991; Lacks and Stalker, 1993; Lanham et al., 1994; Halward et al., 1991, 1992; Kochert et al., 1991; Paik-Ro et al., 1992; Gimenes et al., 2002; Herselman, 2003; Milla-Lewis et al., 2010a, 2010b). Microsatellites or simple sequence repeats (SSRs), the tandem repeats of short (2-6 bp) DNA sequences distributed abundantly throughout eukaryotic genomes, are often highly polymorphic, multi-allelic, and amenable to high-throughput genotyping, making them the markers of choice in diversity assessment, construction of linkage maps, and for assessing marker-trait association (Litt and Lutty 1989). At present, $>1000$ SSR markers are available in peanut (Hopkins et al., 1999; He et al., 2003; Ferguson et al., 2004b; Moretzsohn et al., 2005; Palmieri et al., 2005; Gimenes et al., 2007; Proite et al., 2007; Cuc et al., 2008), some of which have been used to assess genetic diversity in peanut germplasm, including wild species (Hopkins et al., 1999; Moretzsohn et al., 2004; Krishna et al., 2004; Freitas et al., 2007; Tang et al., 2007; Naito et al., 2008; Barkley et al., 2007; Jiang et al., 2007; Mace et al., 2006, 2007; Kottapalli et al., 2007) and construction of genetic maps for the diploid AA- and $\mathrm{BB}$-genomes as well as the AABBgenome (Moretzsohn et al., 2005, 2009; Varshney et al., 2008; Hong et al., 2008; Khedikar et al., 2010), thereby providing tremendous opportunity to identify allelic variation associated with beneficial traits and conduct markerassisted genetic enhancement in peanut. However, the success of any crop improvement program depends on the researcher's ability to detect and exploit the genetic diversity locked in germplasm, including wild relatives that are conserved in various gene banks worldwide. Core collections have been suggested as a gateway to enhance the use of germplasm in crop improvement programs (Frankel, 1984; Brown, 1989). In peanut, both core and mini-core collections have been developed (Holbrook et al., 1993; Upadhyaya et al., 2002, 2003; Holbrook and Dong, 2005; Dwivedi et al., 2008). These subsets have been used to identify new sources of genetic variation for traits of interest (Upadhyaya et al., 2009; Kottapalli et al., 2009) and to assess levels of molecular diversity (Kottapalli et al., 2007).

The objectives of the present investigation were to characterize population structure and diversity in a set of Valencia peanut core collection accessions using SSR markers to identify genetically diverse accessions to broaden the genetic base of Valencia peanut germplasm/cultivars.

\section{MATERIALS AND METHODS}

\section{Plant Materials}

A total of 114 Valencia peanut accessions, including 78 Valencia core collection accessions (Dwivedi et al., 2008), 35 Valencia accessions from global mini-core collection (Upadhyaya et al., 2002), and a control cultivar Gangapuri (Ramamurthy, 1974), constituted the experimental material (Table 1). The majority of accessions (68.4\%) were from South America, followed by Africa (12.2\%), North America (6.1\%), and Asia (6.1\%). Other regions were represented by 1 to $2 \%$.

\section{Isolation of Genomic DNA and SSR Genotyping}

Total genomic DNA was isolated from leaves of 14-d-old seedlings using acetyl trimethyl ammonium bromide-based highthroughput protocol (Mace et al., 2003), with the modification of using a 48-well Retch (Newtown, PA) Mixer Mill MM300 grinder, in which tissue was ground three times at $25 \mathrm{~Hz}$ for 1 min. DNA quantification was done with a ND-1000 Spectrophotometer (Nano Drop Technology, Wilmington, DE) and the samples were diluted to a concentration of $10 \mathrm{ng} \mu \mathrm{L}^{-1}$ for the SSR genotyping. A modified M13-tagged forward, concatenated with a 20-mer M13 oligo (GAC GTT GTA AAA CGA CGGCC) to the $5^{\prime}$ end of each forward primer and normal reverse primer were used for each marker. To facilitate detection, a 20-mer M13 oligo labeled with one of four fluorescent dyes, 6FAM, VIC, NED, or PET, was added to the polymerase chain reaction (PCR) mix to label SSR products of each marker. Initially 100 SSR primers were optimized with four genotypes (PI 493631, PI 565461, PI 576604, and ICG 2738) to identify 52 polymorphic SSR primers (Table 2) that were used for molecular profiling of 114 Valencia accessions. A reference for the PCR conditions used for primer sets obtained from previous studies (Hopkins et al., 1999; Ferguson et al., 2004b; He et al., 2003, 2005; Moretzsohn et al., 2004, 2005) is briefly summarized. Template DNA was amplified using a PTC-225 (MJ Research, Waltham, MA) Peltier thermal cycler with the following conditions: $94^{\circ} \mathrm{C}$ initial denaturation for $15 \mathrm{~min}(1 \mathrm{cycle})$; followed by 10 cycles of denaturation at $94^{\circ} \mathrm{C}$ for $45 \mathrm{~s}$, annealing 65 to $55^{\circ} \mathrm{C}$ touchdown temperature with decrease of $1^{\circ} \mathrm{C}$ per cycle and 
Table 1. Information of 114 Valencia peanut accessions used for the assessment of genetic diversity.

\begin{tabular}{|c|c|c|c|c|c|c|c|}
\hline Serial no. & Code $^{\dagger}$ & Country of origin & Geographic region & Biological status & $\mathrm{NJ}^{\ddagger}$ & $\mathrm{PCoA}^{\S}$ & STRUCTURE \\
\hline 1 & PI 259601 & Australia & Australia & Landrace & I & $\mathrm{B}$ & $1 / 4$ \\
\hline 2 & PI 493536 & Brazil & South America & Advanced cultivar & I & A & $1 / 4$ \\
\hline 3 & PI 259580 & Jamaica & Caribbean & Landrace & I & A & $1 / 3$ \\
\hline 4 & PI 493501 & Brazil & South America & Advanced cultivar & I & $A$ & $1 / 3 / 4 / 5$ \\
\hline 5 & Pl 497447 & Bolivia & South America & Landrace & I & $A$ & $1 / 3$ \\
\hline 6 & PI 602494 & Argentina & South America & Landrace & I & $A$ & $1 / 2$ \\
\hline 7 & PI 493630 & Paraguay & South America & Advanced cultivar & I & A & $1 / 2 / 3 / 4 / 5$ \\
\hline 8 & PI 493518 & Brazil & South America & Advanced cultivar & I & B & $1 / 3 / 4 / 5$ \\
\hline 9 & PI 576604 & Bolivia & South America & Unknown & I & $\mathrm{B}$ & $1 / 2 / 4 / 5$ \\
\hline 10 & PI 493446 & Paraguay & South America & Advanced cultivar & I & $A$ & $1 / 3 / 4 / 2$ \\
\hline 11 & PI 365564 & Bolivia & South America & Landrace & I & $A$ & $1 / 2$ \\
\hline 12 & PI 406718 & Costa Rica & Central America & Landrace & I & $A$ & $1 / 2 / 3$ \\
\hline 13 & PI 429430 & Zimbabwe & Africa & Landrace & I & $A$ & $1 / 2 / 3$ \\
\hline 14 & PI 475913 & Bolivia & South America & Landrace & I & A & $1 / 2$ \\
\hline 15 & PI 476078 & Brazil & South America & Landrace & I & A & $1 / 2$ \\
\hline 16 & PI 429427 & Zimbabwe & Africa & Landrace & I & $A$ & $1 / 2 / 3 / 4 / 5$ \\
\hline 17 & PI 493344 & Paraguay & South America & Advanced cultivar & III & B & $1 / 2 / 4 / 5$ \\
\hline 18 & PI 493688 & Paraguay & South America & Advanced cultivar & I & $A$ & $3 / 4 / 5$ \\
\hline 19 & PI 476089 & Brazil & South America & Landrace & III & B & $1 / 2 / 3 / 4$ \\
\hline 20 & PI 493339 & Bolivia & South America & Advanced cultivar & I & $A$ & $3 / 4 / 5$ \\
\hline 21 & PI 493458 & Paraguay & South America & Advanced cultivar & $\|$ & $E$ & $1 / 2 / 3 / 4$ \\
\hline 22 & PI 493507 & Brazil & South America & Advanced cultivar & I & $A$ & $4 / 5$ \\
\hline 23 & PI 476079 & Brazil & South America & Landrace & I & $A$ & $1 / 2 / 3$ \\
\hline 24 & PI 476074 & Brazil & South America & Landrace & I & $A$ & $1 / 2 / 3$ \\
\hline 25 & PI 493562 & Brazil & South America & Advanced cultivar & । & $A$ & $2 / 3$ \\
\hline 26 & PI 497459 & Bolivia & South America & Landrace & III & B & $2 / 3 / 4$ \\
\hline 27 & PI 493405 & Paraguay & South America & Advanced cultivar & $\|$ & $E$ & $1 / 2 / 3 / 4 / 5$ \\
\hline 28 & PI 493461 & Brazil & South America & Advanced cultivar & I & $A$ & $3 / 4$ \\
\hline 29 & PI 493865 & Paraguay & South America & Advanced cultivar & $\|$ & $A$ & $1 / 2 / 3 / 4$ \\
\hline 30 & PI 493415 & Paraguay & South America & Advanced cultivar & I & A & $2 / 3 / 4$ \\
\hline 31 & PI 493470 & Brazil & South America & Advanced cultivar & I & A & $1 / 2 / 3$ \\
\hline 32 & PI 315612 & Africa & Africa & Advanced cultivar & $\|$ & $A$ & $1 / 2 / 4$ \\
\hline 33 & PI 493325 & Paraguay & South America & Advanced cultivar & $\|$ & $\mathrm{B}$ & $2 / 4 / 5$ \\
\hline 34 & PI 493340 & Bolivia & South America & Advanced cultivar & I & A & $2 / 3 / 4$ \\
\hline 35 & PI 493624 & Paraguay & South America & Advanced cultivar & I & $A$ & $1 / 2 / 3 / 4$ \\
\hline 36 & PI 493810 & Brazil & South America & Advanced cultivar & I & A & $1 / 2 / 3$ \\
\hline 37 & PI 501985 & Peru & South America & Landrace & $\|$ & $A$ & $1 / 2 / 3$ \\
\hline 38 & PI 338337 & Venezuela & South America & Advanced cultivar & III & B & $2 / 3 / 4 / 5$ \\
\hline 39 & Breedingline & USA & North America & New breeding line & I & $A$ & $3 / 4 / 5$ \\
\hline 40 & PI 493382 & Paraguay & South America & Advanced cultivar & I & $A$ & $2 / 3 / 5$ \\
\hline 41 & PI 493360 & Paraguay & South America & Advanced cultivar & I & $A$ & $1 / 2 / 3$ \\
\hline 42 & PI 493523 & Brazil & South America & Advanced cultivar & I & $A$ & $2 / 4$ \\
\hline 43 & PI 475925 & Bolivia & South America & Landrace & III & B & $1 / 2 / 3 / 4 / 5$ \\
\hline 44 & PI 494019 & Brazil & South America & Advanced cultivar & $\|$ & $A$ & $2 / 3 / 4$ \\
\hline 45 & PI 493565 & Brazil & South America & Advanced cultivar & $\|$ & $A$ & $2 / 3 / 4$ \\
\hline 46 & PI 493584 & Brazil & South America & Advanced cultivar & I & A & $2 / 3$ \\
\hline 47 & PI 536300 & Uruguay & South America & Landrace & III & A & $1 / 2$ \\
\hline 48 & PI 493660 & Paraguay & South America & Advanced cultivar & I & $A$ & $2 / 3 / 4 / 5$ \\
\hline 49 & PI 536307 & Uruguay & South America & Landrace & III & $B$ & $2 / 3$ \\
\hline 50 & PI 493373 & Paraguay & South America & Advanced cultivar & I & $A$ & $2 / 3 / 4 / 5$ \\
\hline 51 & PI 497642 & Ecuador & South America & Landrace & I & A & $1 / 2 / 3 / 4$ \\
\hline 52 & PI 493612 & Bolivia & South America & Advanced cultivar & I & A & $1 / 2 / 3 / 5$ \\
\hline 53 & PI 493484 & Brazil & South America & Advanced cultivar & I & A & $1 / 2 / 3 / 4$ \\
\hline 54 & PI 493816 & Paraguay & South America & Advanced cultivar & I & A & $1 / 2$ \\
\hline 55 & PI 493566 & Brazil & South America & Advanced cultivar & । & $A$ & $2 / 3 / 4$ \\
\hline
\end{tabular}


Table 1. Continued.

\begin{tabular}{|c|c|c|c|c|c|c|c|}
\hline Serial no. & Code $^{\dagger}$ & Country of origin & Geographic region & Biological status & $\mathrm{NJ}^{\ddagger}$ & $\mathrm{PCoA}^{\S}$ & STRUCTUREף \\
\hline 56 & PI 493451 & Paraguay & South America & Advanced cultivar & I & $A$ & $1 / 2 / 3$ \\
\hline 57 & PI 501269 & Peru & South America & Landrace & I & A & $1 / 2 / 3$ \\
\hline 58 & PI 536121 & Brazil & South America & Breeding material & । & A & $3 / 4 / 5$ \\
\hline 59 & PI 493514 & Brazil & South America & Advanced cultivar & । & $A$ & $2 / 3 / 4 / 5$ \\
\hline 60 & PI 468208 & Bolivia & South America & Advanced cultivar & । & $A$ & $1 / 2$ \\
\hline 61 & PI 493666 & Paraguay & South America & Advanced cultivar & । & $A$ & $1 / 2 / 3 / 4$ \\
\hline 62 & PI 493381 & Paraguay & South America & Unknown & । & A & $1 / 2 / 3$ \\
\hline 63 & PI 502023 & Peru & South America & Unknown & । & A & $1 / 2 / 3$ \\
\hline 64 & PI 475921 & Bolivia & South America & Landrace & । & A & $1 / 2 / 3$ \\
\hline 65 & PI 501293 & Peru & South America & Breeding material & I & A & $1 / 2 / 3 / 5$ \\
\hline 66 & PI 306361 & Israel & Asia & Advanced cultivar & । & $A$ & $2 / 3$ \\
\hline 67 & PI 493629 & Paraguay & South America & Advanced cultivar & । & A & $1 / 2$ \\
\hline 68 & PI 493442 & Paraguay & South America & Advanced cultivar & । & A & $2 / 3 / 4$ \\
\hline 69 & PI 407451 & Ecuador & South America & Unknown & । & A & $1 / 2 / 3$ \\
\hline 70 & PI 390432 & Ecuador & South America & Landrace & । & A & $1 / 2 / 3$ \\
\hline 71 & PI 409037 & Zimbabwe & Africa & Landrace & । & A & $1 / 2 / 3 / 4$ \\
\hline 72 & Grif13802 & Ecuador & South America & Unknown & III & B & $2 / 3 / 4$ \\
\hline 73 & PI 599612 & Bolivia & South America & Advanced cultivar & । & B & $3 / 4 / 5$ \\
\hline 74 & PI 508278 & USA & North America & Breeding material & । & A & $2 / 3 / 4 / 5$ \\
\hline 75 & PI 493521 & Brazil & South America & Advanced cultivar & । & B & $2 / 3 / 4$ \\
\hline 76 & PI 314980 & Russia & Europe & Landrace & I & B & $2 / 3 / 4$ \\
\hline 77 & PI 468225 & Bolivia & South America & Landrace & - & A & $2 / 3 / 4$ \\
\hline 78 & PI 493631 & Paraguay & South America & Advanced cultivar & । & B & $1 / 2 / 3 / 4$ \\
\hline 79 & ICG 5609 & Sri Lanka & Asia & Landrace & - & $E$ & $3 / 4 / 5$ \\
\hline 80 & ICG 8517 & Bolivia & South America & Landrace & $\|$ & $\mathrm{B}$ & $3 / 4$ \\
\hline 81 & ICG 15042 & UK & Europe & Other & IV & $A$ & $3 / 4 / 5$ \\
\hline 82 & ICG 6646 & UK & Europe & Landrace & IV & B & $1 / 2 / 3 / 4 / 5$ \\
\hline 83 & ICG 5475 & Kenya & Africa & Other & - & $E$ & $1 / 3 / 4 / 5$ \\
\hline 84 & ICG 7181 & India & Asia & Landrace & $\|$ & $E$ & $3 / 4$ \\
\hline 85 & ICG 6022 & Sudan & Africa & Advanced cultivar & $\|$ & B & $3 / 4$ \\
\hline 86 & ICG 10092 & Zimbabwe & Africa & Landrace & - & B & $1 / 2 / 3 / 4 / 5$ \\
\hline 87 & ICG 6888 & Brazil & South America & Breeding material & IV & $\mathrm{B}$ & $3 / 4 / 5$ \\
\hline 88 & ICG 8106 & Peru & South America & Landrace & IV & B & $3 / 4 / 5$ \\
\hline 89 & ICG 332 & Brazil & South America & Landrace & IV & B & $1 / 2 / 3 / 4 / 5$ \\
\hline 90 & ICG 13856 & Uruguay & South America & Landrace & IV & $\mathrm{B}$ & $2 / 3 / 4 / 5$ \\
\hline 91 & ICG 397 & USA & North America & Breeding material & IV & B & $2 / 4 / 5$ \\
\hline 92 & ICG 9315 & USA & North America & Breeding material & IV & B & $1 / 2 / 3 / 4 / 5$ \\
\hline 93 & ICG 3681 & USA & North America & Other & IV & B & $3 / 2 / 5$ \\
\hline 94 & ICG 14127 & Zaire & Africa & Other & IV & B & $1 / 2 / 4 / 5$ \\
\hline 95 & ICG 14630 & Brazil & South America & Landrace & IV & B & $1 / 2 / 4 / 5$ \\
\hline 96 & ICG 10566 & Congo & Africa & Other & IV & B & $3 / 4 / 5$ \\
\hline 97 & ICG 115 & India & Asia & Landrace & IV & C & $1 / 4 / 5$ \\
\hline 98 & ICG 13858 & Uruguay & South America & Landrace & V & C & $3 / 4 / 5$ \\
\hline 99 & ICG 10890 & Peru & South America & Landrace & V & C & $1 / 3 / 4 / 5$ \\
\hline 100 & ICG 11144 & Argentina & South America & Breeding material & V & C & $3 / 4 / 5$ \\
\hline 101 & ICG 1399 & Malawi & Africa & Breeding material & V & C & $3 / 5$ \\
\hline 102 & ICG 15309 & Brazil & South America & Landrace & V & C & $2 / 3 / 5$ \\
\hline 103 & ICG 4670 & Sudan & Africa & Other & V & C & $2 / 3 / 4 / 5$ \\
\hline 104 & ICG 5221 & Argentina & South America & Landrace & V & $\mathrm{C}$ & $3 / 4 / 5$ \\
\hline 105 & ICG 1274 & India & Asia & Other & V & $\mathrm{D}$ & $3 / 4 / 5$ \\
\hline 106 & ICG 3673 & Korea & Asia & Landrace & V & $\mathrm{D}$ & $3 / 4 / 5$ \\
\hline 107 & ICG 14106 & Zaire & Africa & Other & V & $\mathrm{D}$ & $3 / 4 / 5$ \\
\hline 108 & ICG 1142 & Benin & Africa & Breeding material & V & C & $3 / 4 / 5$ \\
\hline 109 & ICG 10554 & Argentina & South America & Breeding material & V & C & $1 / 3 / 4 / 5$ \\
\hline 110 & ICG 10474 & Cuba & Caribbean & Advanced cultivar & V & - & $2 / 3 / 5$ \\
\hline
\end{tabular}


Table 1. Continued.

\begin{tabular}{|c|c|c|c|c|c|c|c|}
\hline Serial no. & Code $^{\dagger}$ & Country of origin & Geographic region & Biological status & $\mathrm{NJ}^{\ddagger}$ & $\mathrm{PCoA}^{\S}$ & STRUCTURE \\
\hline 111 & ICG 6201 & Cuba & Caribbean & Landrace & V & $\mathrm{D}$ & $3 / 4 / 5$ \\
\hline 112 & ICG 297 & USA & North America & Advanced cultivar & V & B & $3 / 4 / 5$ \\
\hline 113 & ICG 14710 & Cameroon & Africa & Landrace & III & $\mathrm{B}$ & $3 / 4 / 5$ \\
\hline 114 & ICG 2738 & India & Asia & Advanced cultivar & $\|$ & A & $1 / 3 / 4 / 5$ \\
\hline
\end{tabular}

† Plant Introduction (PI) accession code refers to U.S. Valencia Mini-core collection; ICG refers to groundnut from ICRISAT gene bank.

‡ Classification by neighbor-joining (NJ) tree using Nei's coefficient based on 52 simple sequence repeat (SSR) markers.

$\S$ Classification based on principal coordinate analysis (PCoA) based on 52 SSR markers.

" Classification based on STRUCTURE analysis based on 52 SSR markers.

extension at $72^{\circ} \mathrm{C}$ for $30 \mathrm{~s}$, followed by 35 cycles of $94^{\circ} \mathrm{C}$ for 45 $\mathrm{s}, 55^{\circ} \mathrm{C}$ for $60 \mathrm{~s}$, extension at $72^{\circ} \mathrm{C}$ for $30 \mathrm{~s}$, and a final extension at $72^{\circ} \mathrm{C}$ for $10 \mathrm{~min}$. The PCR amplifications were achieved in a volume of $5 \mu \mathrm{L}$, containing $1 \times$ PCR buffer $(10 \mathrm{mM}$ Tris- $\mathrm{HCl}$, $\mathrm{pH} 8.3,50 \mathrm{mM} \mathrm{KCl}, 1.5 \mathrm{mM} \mathrm{MgCl} 2$ ), $0.2 \mathrm{mM}$ of each dNTP, 0.25 units Hotstart Taq DNA polymerase (Qiagen Inc., Valencia, CA), 5 pmol of each M13-tagged forward and normal reverse primer, 0.02 pmol of fluorescently labeled 20-mer M13 primer (labeled with either 6FAM, VIC, NED, or PET), and $10 \mathrm{ng}$ of template genomic DNA. The PCR-amplified products $(1 \mu \mathrm{L}$ each) were multiplexed into a mixture of Hi-Di formamide (10 $\mu \mathrm{L}$ ) containing an internal standard GeneScan-500 LIZ (Applied Biosystems, Foster City, CA), denatured at $94^{\circ} \mathrm{C}$ for $5 \mathrm{~min}$, and separated by capillary electrophoresis on an ABI Prism 3130 DNA Analyzer (Applied Biosystems, Foster City, CA) according to manufacturer's instructions. Fragments were called using Gene Mapper V4.0 software (Applied Biosystems Inc., Foster City, CA).

\section{Data Analysis}

The amplification products were scored as present (1) or absent (0) for each of the accessions to compute the binary data matrix that was analyzed using NTSYS-PC version 2.2 (Numerical Taxonomy and System) (Rohlf, 2000). A genetic distance matrix was developed and subsequently used to construct dendrograms based on the neighbor-joining (NJ) clustering method and visualized using the tree program of the NTSYS-PC version 2.2. To measure the goodness of fit for cluster analysis, a cophenetic correlation value between the original similarity matrix and the cophenetic matrix was compared using the MXCOMP procedure of NTSYS-PC version 2.2. The significance of correlation between the matrices was tested using the normalized Mantel $Z$-statistics (Mantel, 1967). The binary data were also subjected to principal coordinate analysis (PCoA) to partition the variance using NTSYS-PC version 2.2 and the first three components were plotted into MOD three-dimensional scatter plots.

Power Marker V 3.25 (Liu and Muse, 2005) was used to detect polymorphic information content (PIC) and gene diversity. The PIC measures allelic diversity at a locus (Botstein et al., 1980), while gene diversity is defined as the probability that two randomly chosen alleles from the population are different (Nei, 1973). A distance matrix was generated based on the number of shared alleles between different countries and unrooted NJ tree was constructed. Pairwise comparisons of genetic distances among the accessions were calculated using Nei's formula (Nei, 1972).

The model-based program, STRUCTURE version 2.3.1 (http://pritch.bsd.uchicago.edu/software/structure_v.2.3.1.html [verified 15 Feb. 2011]) was used to determine $K$, the number of structured groups (Pritchard et al., 2000; Falush et al., 2003, 2007). The ancestry model with admixture was chosen and using the correlated allele frequency option, multiple runs of STRUCTURE were performed by setting $K$ from one to 10 groups. The length of burn-in was set at 10,000 followed by 100,000 iterations, and each run was replicated 10 times. Runs with the highest $L n \mathrm{P}(\mathrm{D})$ probability were considered for each $K$ and graphical outputs were visualized to determine the most appropriate number of clusters.

\section{RESULTS}

\section{Molecular Marker Diversity}

The 52 SSRs detected a total of 683 alleles, with an average of 13 alleles per primer pair (Table 2). The number of alleles ranged from two (TC4H2) to 28 (PM3), with large number of SSRs detecting 11 to 15 alleles (Fig. 1). Amplicon sizes ranged from 100 (TC11E4) to 499 bp (Ah193). The PIC ranged from 0.129 (gi716) to 0.361 (pPGPseq4D4) with a mean of 0.270 , which revealed low to moderate levels of polymorphism. Gene diversity ranged from 0.1427 to 0.4743 with a mean of 0.335 , indicating a moderate level of diversity in the Valencia peanut germplasm. Based on estimates of gene diversity, the most informative markers were pPGPseqD4, pPGPseq2A6, pPGPseq7G2, TC9H8, and PM238, while the least informative were gi716 and pPGSseq17E3.

\section{Geographical Diversity}

The 114 accessions included in this study originated from 27 countries belonging to different geographic regions of the world (Table 1). To understand the geographic diversity and underlying genetic diversity of the Valencia genotypes, the binary data from SSR markers were used to generate a distance matrix based on number of shared alleles between different countries and an unrooted NJ tree was constructed. The clustering based on shared SSR alleles between countries could be useful in understanding pattern of dispersal of the Valencia germplasm across the world from its source of origin in South America. The 27 countries were grouped into five clusters (Fig. 2). Cluster 1 from Malawi and Benin shared common alleles with Korea and Cuba. Kenya, Sri Lanka, and Venezuela shared common alleles with the Congo in Cluster 2. Accessions from Costa Rica, Jamaica, and Australia shared common alleles with countries from Africa, Russia, and Israel in Cluster 3. The accessions from Cameroon separated 
Table 2. List of simple sequence repeat primers, number of alleles, size range, and summary of genetic diversity analysis components analyzed.

\begin{tabular}{|c|c|c|c|c|c|}
\hline Marker & $\begin{array}{l}\text { No. of } \\
\text { alleles }\end{array}$ & $\begin{array}{l}\text { Size } \\
\text { range }\end{array}$ & Availability $^{\dagger}$ & $\begin{array}{c}\text { Gene } \\
\text { diversity }\end{array}$ & $\mathrm{PIC}^{\ddagger}$ \\
\hline & & $\mathrm{bp}$ & & & \\
\hline Ah41 & 12 & 277-329 & 1.0000 & 0.4221 & 0.3295 \\
\hline Ah193 & 22 & $292-499$ & 0.9825 & 0.4130 & 0.3231 \\
\hline Ah229 & 7 & 121-238 & 0.9912 & 0.3715 & 0.2966 \\
\hline Ah4_20 & 16 & $220-227$ & 0.9912 & 0.3297 & 0.2711 \\
\hline Ah4_26 & 13 & 170-296 & 0.9474 & 0.3928 & 0.3141 \\
\hline gi716 & 11 & $145-347$ & 0.9912 & 0.1427 & 0.1298 \\
\hline PM3 & 28 & $187-255$ & 0.9825 & 0.3740 & 0.2989 \\
\hline PM32 & 14 & $106-224$ & 1.0000 & 0.3805 & 0.3048 \\
\hline PM35 & 27 & 121-182 & 1.0000 & 0.3671 & 0.2955 \\
\hline PM36 & 19 & $117-228$ & 1.0000 & 0.3342 & 0.2725 \\
\hline PM45 & 14 & 103-163 & 0.8772 & 0.3773 & 0.3012 \\
\hline PM65 & 23 & 214-265 & 0.9825 & 0.4332 & 0.3363 \\
\hline PM137 & 11 & $148-223$ & 0.9298 & 0.3356 & 0.2660 \\
\hline PM183 & 16 & 124-174 & 0.9912 & 0.3652 & 0.2888 \\
\hline PM188 & 12 & $105-140$ & 0.9912 & 0.3366 & 0.2753 \\
\hline PM204 & 19 & $211-251$ & 0.9912 & 0.4160 & 0.3246 \\
\hline PM238 & 22 & $162-221$ & 0.9825 & 0.4504 & 0.3473 \\
\hline PM375 & 14 & $117-178$ & 0.9912 & 0.2785 & 0.2322 \\
\hline pPGPseq1B9 & 15 & $278-320$ & 1.0000 & 0.3685 & 0.2928 \\
\hline pPGPseq2A5 & 11 & 103-180 & 1.0000 & 0.2024 & 0.1807 \\
\hline pPGPseq2A6 & 14 & 200-288 & 1.0000 & 0.4662 & 0.3557 \\
\hline pPGPseq3C2 & 19 & $297-340$ & 0.9649 & 0.4333 & 0.3351 \\
\hline pPGPseq3E10 & 7 & 119-235 & 0.9912 & 0.3146 & 0.2540 \\
\hline pPGPseq4D4 & 12 & $200-253$ & 0.9905 & 0.4743 & 0.3616 \\
\hline pPGPseq4H11 & 14 & 232-296 & 1.0000 & 0.2041 & 0.1828 \\
\hline pPGPseq5D5 & 6 & 270-301 & 0.9912 & 0.3502 & 0.2831 \\
\hline pPGPseq6B8 & 8 & $185-264$ & 0.9912 & 0.4150 & 0.3270 \\
\hline pPGPseq7G2 & 9 & 233-267 & 0.9561 & 0.4638 & 0.3556 \\
\hline pPGPseq7H6 & 18 & $146-317$ & 1.0000 & 0.4096 & 0.3220 \\
\hline pPGPseq8E12 & 8 & 199-221 & 0.9035 & 0.4308 & 0.3346 \\
\hline pPGSseq9F1 & 11 & 243-284 & 0.9825 & 0.3357 & 0.2747 \\
\hline pPGSseq13E11 & 8 & $145-338$ & 0.9912 & 0.1836 & 0.1629 \\
\hline pPGSseq16G8 & 17 & $211-477$ & 0.8509 & 0.3317 & 0.2727 \\
\hline pPGSseq17E3 & 5 & 194-217 & 1.0000 & 0.1546 & 0.1409 \\
\hline pPGSseq18C5 & 7 & $138-239$ & 0.8509 & 0.2246 & 0.1910 \\
\hline pPGSseq19A5 & 12 & $138-281$ & 0.9825 & 0.1953 & 0.1681 \\
\hline pPGSseq19D6 & 5 & $306-365$ & 0.8509 & 0.2017 & 0.1661 \\
\hline pPGSseq19G7 & 19 & 129-198 & 0.9825 & 0.3204 & 0.2621 \\
\hline TC1E6 & 16 & $141-173$ & 1.0000 & 0.3723 & 0.2966 \\
\hline TC3G1 & 13 & 184-279 & 0.9825 & 0.3440 & 0.2732 \\
\hline TC4D9 & 16 & $128-257$ & 0.9825 & 0.3538 & 0.2843 \\
\hline TC4F12 & 8 & $235-256$ & 1.0000 & 0.2868 & 0.2452 \\
\hline TC4G10 & 10 & $141-158$ & 1.0000 & 0.2968 & 0.2484 \\
\hline TC4H2 & 2 & $122-138$ & 1.0000 & 0.1668 & 0.1480 \\
\hline TC6E1 & 15 & 126-189 & 0.9825 & 0.2031 & 0.1789 \\
\hline TC6H3 & 12 & $121-253$ & 0.9825 & 0.2801 & 0.2343 \\
\hline TC7E4 & 18 & $110-175$ & 0.9825 & 0.2893 & 0.2406 \\
\hline TC9F10 & 7 & 264-298 & 0.9825 & 0.3513 & 0.2788 \\
\hline ТС9H8 & 12 & 234-282 & 1.0000 & 0.4417 & 0.3380 \\
\hline TC11A2 & 10 & 155-295 & 1.0000 & 0.3526 & 0.2876 \\
\hline TC11A4 & 12 & $186-217$ & 0.7807 & 0.3598 & 0.2920 \\
\hline TC11E4 & 7 & 100-116 & 1.0000 & 0.3264 & 0.2696 \\
\hline Mean & 13.1346 & & 0.9727 & 0.3351 & 0.2701 \\
\hline Total & 683 & & & & \\
\hline
\end{tabular}

† Percentage available data.

‡ PIC, polymorphism information content.

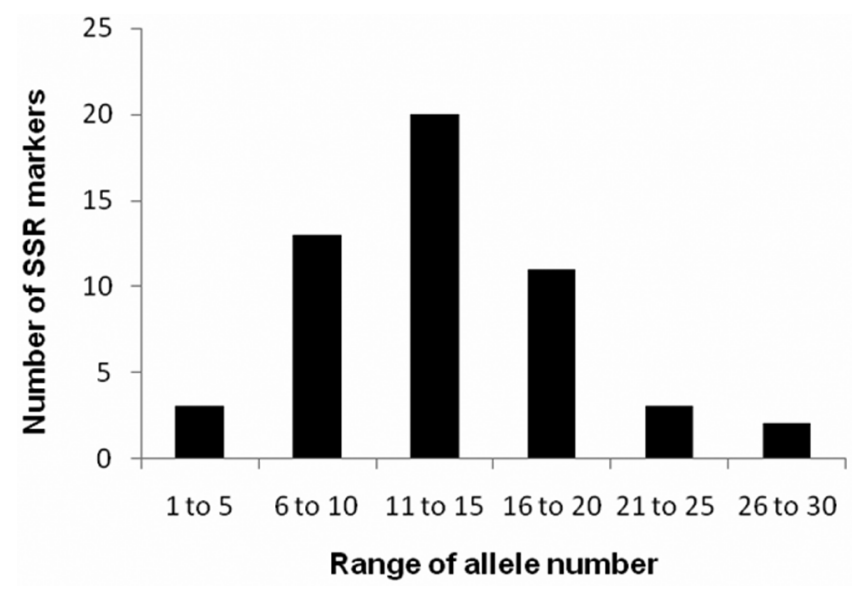

Figure 1. Distribution of allele numbers of 52 polymorphic simple sequence repeat (SSR) loci in 114 Valencia peanut genotypes representing global Valencia peanut germplasm.

as an individual group in Cluster 4. Accessions from Brazil, Paraguay, Bolivia, Ecuador, Peru, Argentina, and Uruguay grouped in Cluster 5 and shared common alleles with those accessions from the United States, United Kingdom, Zaire, Sudan, Zimbabwe, and India.

\section{Neighbor-joining Clustering}

Six hundred eighty-three alleles from 52 SSR loci were used to estimate pairwise genetic distances among the accessions based on Nei's coefficient. A high correlation $(r$ $=0.88$ ) between the cophenetic matrix and the original matrix reveals a good fit of the genotype clustering. The average genetic distance was 0.631 , representing high variability among the Valencia peanut accessions. A dendrogram constructed using NJ method based on Nei's genetic distance grouped 114 accessions into five distinct clusters, with two major groups (Fig. 3 and Table 1). Group A was the largest cluster, with 72 accessions originating mostly from South America, with few from Africa, North and Central America, Asia, and Caribbean regions. Further, two clusters (I and II) in this group separated at a genetic distance of 0.91. Group B consisted of 38 accessions from diverse regions (Africa, North and South America, Asia, Europe, and the Caribbean), separated into three clusters (III, IV, and V). Furthermore, four accessions, ICG 5609, ICG 5475, PI 468225, and ICG 10092, were not the part of either of the two major groups.

\section{Principal Coordinate Analysis}

The molecular genetic diversity of the SSR loci was also subjected to PCoA to validate the clustering pattern. The first three components were plotted into MOD threedimensional scatterplots (Fig. 4). Principal Component (PC) 1 and PC 2 accounted for 54.4 and $5.3 \%$ of the total variation, respectively. The first three components together explained $62.4 \%$ of the total variation. The accessions were clustered into five distinct groups, A, B, C, D, and E (Table 


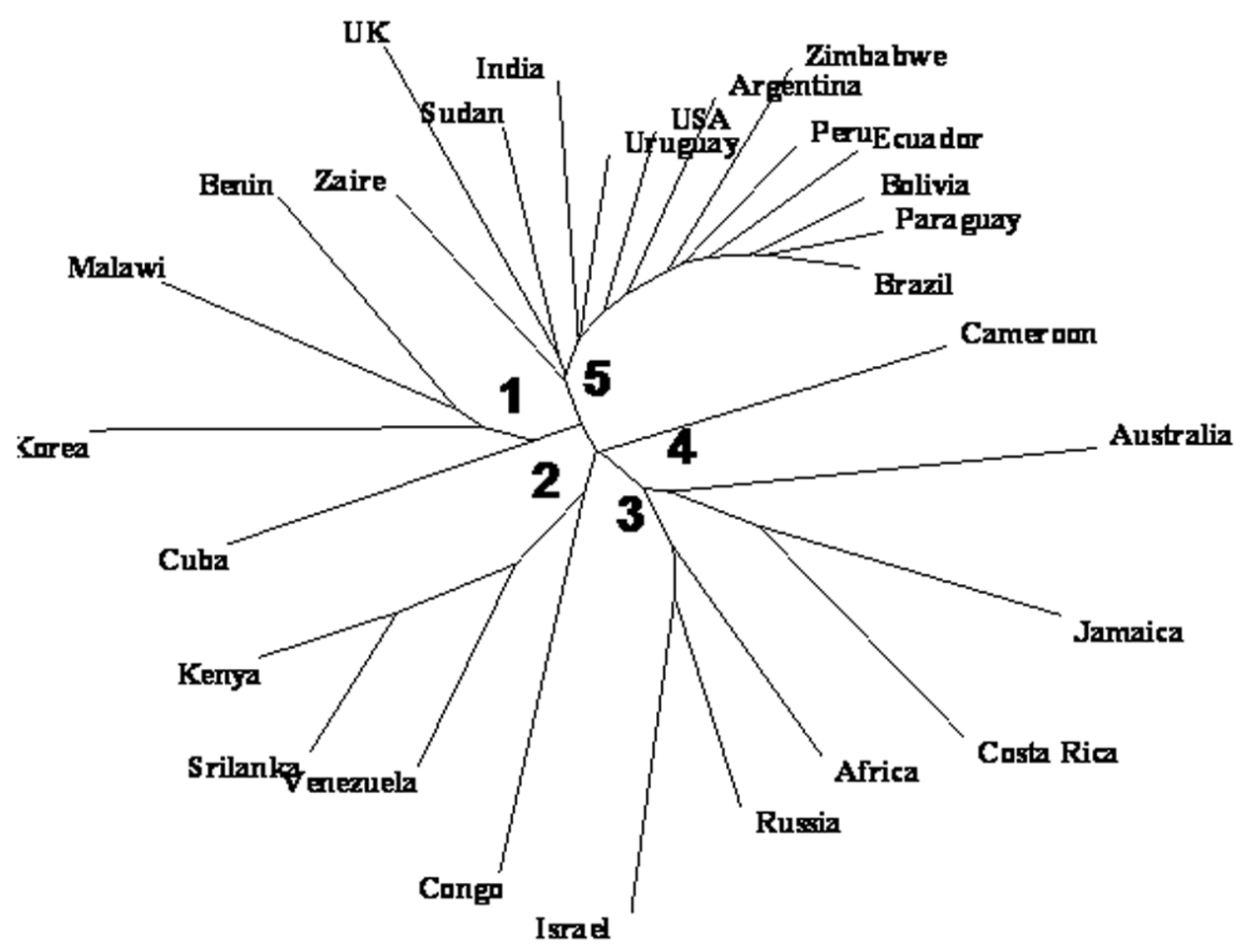

Figure 2. Unrooted neighbor-joining tree constructed based on number of shared alleles among different source countries.

1 and Fig. 4), except for ICG 10474, an advanced cultivar from Cuba, which was placed far apart from the rest of the accessions. Groups A and B consisted of 63 and 31 accessions, respectively. Group A consisted of 20 accessions from Brazil and 16 accessions from Paraguay, mostly advanced cultivars. It also had representation from Bolivia (nine accessions), Peru (four accessions), Ecuador (two accessions), Zimbabwe (three landraces), United States (two accessions), one cultivar each from India, Israel, and in Africa, and one each from the United Kingdom, Uruguay, Costa Rica, and Argentina. Cluster B consisted of 19 accessions from South America, (six from Brazil, five from Bolivia, three from Paraguay, two from Uruguay, and a single accession each from Ecuador, Peru, and Venezuela), four accessions from the United States, five accessions from Africa, one each from Cameroon, Congo, Sudan, Zaire, and Zimbabwe, and single accessions from Australia, United Kingdom, and Russia. Group C consisted of 10 accessions, six from South America and one each from India, Benin, Malawi, and Sudan. Four accessions from India, Korea, Zaire, and Cuba formed the minor Group D. Two cultivars from Paraguay showed close affinity toward accessions from Sri Lanka, Kenya, and India, and grouped together in Cluster E.

\section{STRUCTURE Analysis}

STRUCTURE 2.3.1 was used to find appropriate numbers of clusters with no prior information about the geographical origin of the genotypes. Population STRUCTURE analysis revealed that $\mathrm{LnP}$ (D) estimates increased from $K=1$ to $K=5$ and then began to plateau, suggesting the genetic diversity should be structured into five subpopulations. The overall proportion of membership in each of the five clusters has $15.2 \%$ (1-red), 30.7\% (2-green), 13.3\% (3-blue), 25.2\% (4-yellow), and 15.6\% (5-pink) (Fig. 5 and Table 1). Admixtures were observed among all the clusters, with eight accessions (PI 493630, PI 429427, PI 493405, PI 475925, ICG 6646, ICG 10092, ICG 332, and ICG 9315) forming admixtures in all five clusters (Table 1). STRUCTURE analysis also identified the two major clusters, obtained by NJ clustering (Fig. 3 and Fig. 5). The five $K$ s structuration also corresponded to the genetic clusters revealed by the PCoA plots (Fig. 4). However, the structuring was not related to the geographic origin of the populations (Table 1).

\section{DISCUSSION}

Cultivated peanut has a narrow genetic base (Knauft and Gorbet, 1989). In the United States, the few Valencia peanut cultivars that are commercially grown were primarily based on selections from plant introductions (Isleib et al., 2001). Identification of genetically diverse germplasm with beneficial traits and their use in peanut breeding is the key to broaden the genetic base of peanut cultivars. The researchers at New Mexico State University are engaged in a systematic evaluation of Valencia germplasm to identify genetically diverse germplasm with beneficial traits. To this end, a core collection of 78 Valencia accessions (Dwivedi et al., 2008) and 36 diverse Valencia germplasm accessions from global mini-core collection (Upadhyaya et al., 2002) were assessed for genetic diversity in this study. Characterization of genetic 


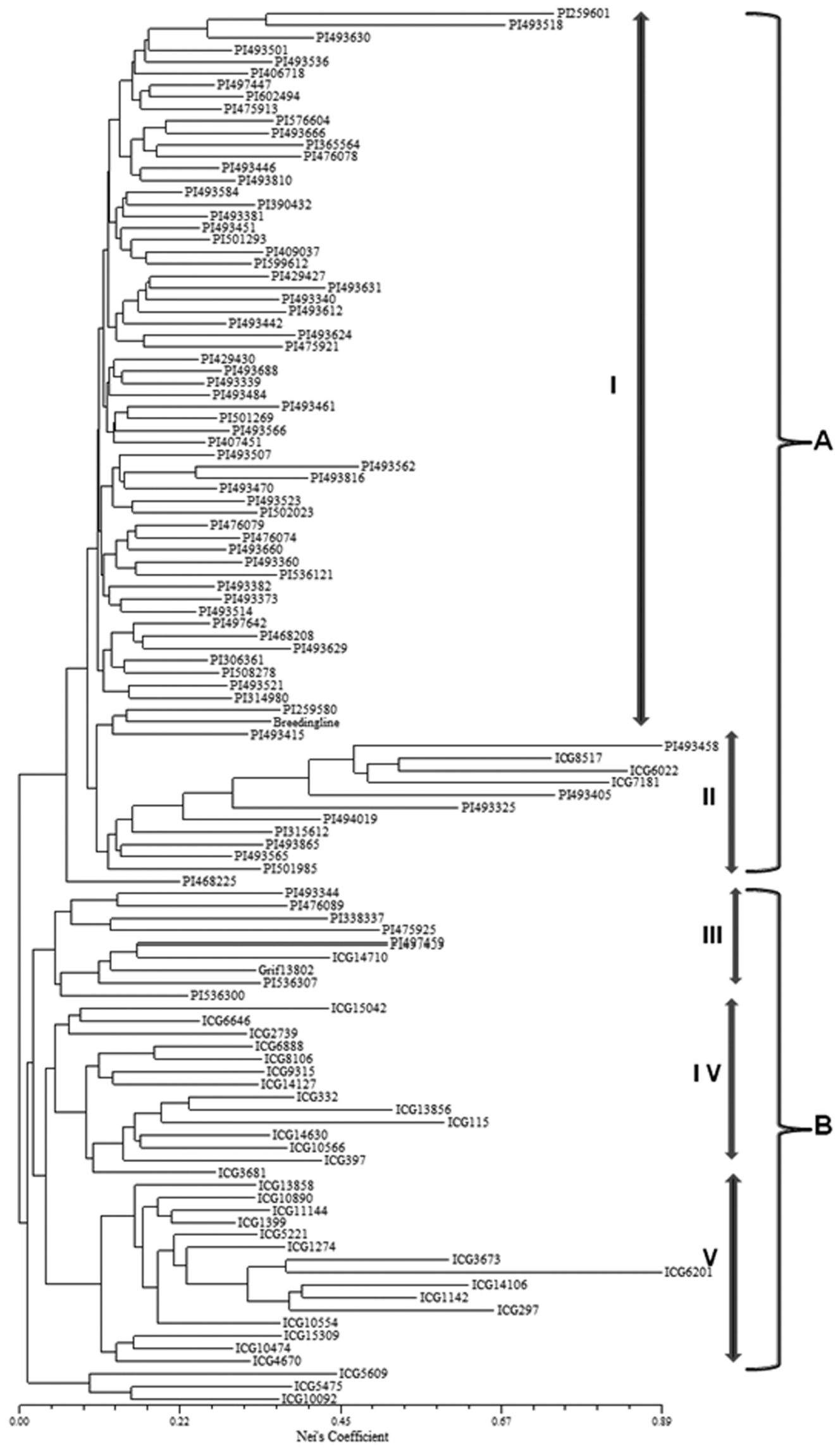

Figure 3. Neighbor-joining tree constructed using Nei's coefficient for 114 Valencia peanut genotypes from global Valencia peanut germplasm. Two main groups (A and B) with five clusters (I-V) are depicted. Source of their accessions: PI = U.S. Valencia Core; ICG = ICRISAT gene bank. 


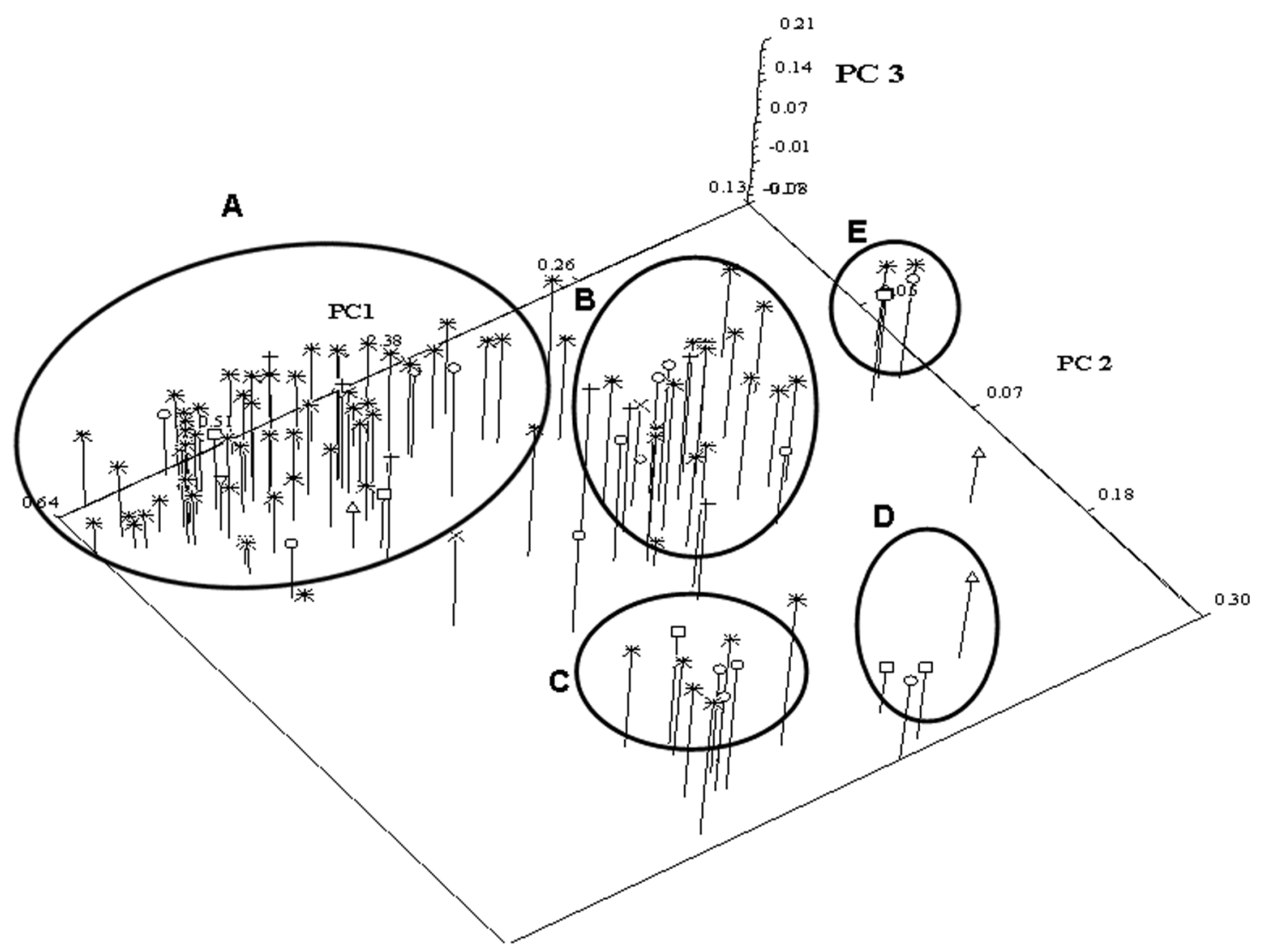

Figure 4. Principal coordinate analysis for 114 Valencia peanut accessions based on 52 simple sequence repeat markers. Two main groups, $A$ and $B$, were identified. Each geographical region is identified by the following symbol: $O, A$ frica; $\square$, Asia; $\nabla$, Australia; $\triangle$, Caribbean; $\nabla$, Central America; X, Europe; +, North America; *, South America. PC, principal component.

diversity in core or mini-core subsets of germplasm should lead to identification of genetically diverse germplasm for use in breeding and genomic applications.

Fifty-eight SSR s loci in the present study detected 683 alleles, averaging 13 alleles per locus, similar to Barkley et al. (2007). Other studies reported large variations in detecting the average number of alleles, ranging from three (Mace et al., 2007) to 31 alleles per locus (Krishna et al., 2004). The number of alleles detected per primer generally depends on the number of tandem repeat units, population size, and also on the type of electrophoresis employed to resolve the amplified fragments (Hwang et al., 2008). The moderate diversity detected by mean PIC (0.27) and gene diversity (0.335) in this set of Valencia germplasm is comparatively lower than those reported for the United States mini-core collection accessions, which represents all four market types (Kottapalli et al., 2007). This result is not surprising given the fact that we examined a single market type.

The SSR data were further used to compute the matrix based on shared alleles between accessions from different countries and the resulting $\mathrm{NJ}$ clustering revealed some interesting groupings (Fig. 2). Cluster 5 had common alleles representing South American countries such as Paraguay, Bolivia, Ecuador, Peru, and Brazil. In our data set, 22.8\% of accessions were from Brazil. This would be expected, as
Valencia genotypes are widely distributed in South America and predominantly in five centers of diversity, with Brazil being one of the important centers of diversity for Valencia peanut (Ferguson et al., 2004a; Kottapalli et al., 2007). In the entire data set a single genotype was available from Venezuela (PI 338337). Interestingly, this accession was placed in Cluster 2, which is devoid of any additional accessions from South American countries. Mostly, accessions from Asia, Africa, the Caribbean, Central America, and Europe did not share common alleles with Cluster 5, which is dominated by accessions from South America. Our findings are similar to those reported by Ferguson et al. (2004a), who concluded that for the botanical varieties of peanut, landraces from Asia and Africa were more closely related to each other than those from South America. The NJ cluster analysis and PCoA estimated the genetic diversity among the accessions and revealed the presence of two major groups. Group A (64.0\% of accessions) in the NJ analysis and Group A (55.6\% of accessions) in the PCoA analysis (Fig. 3 and 4), both largest clusters, comprised accessions mainly from the South American region. Group B consisted of accessions from most of the geographical regions of the world. The two major consensus groups obtained by $\mathrm{NJ}$ and PCoA approaches were in accordance to the consensus clustering reported by Mace et al. $(2006,2007)$ with multidimensional scaling plots and 


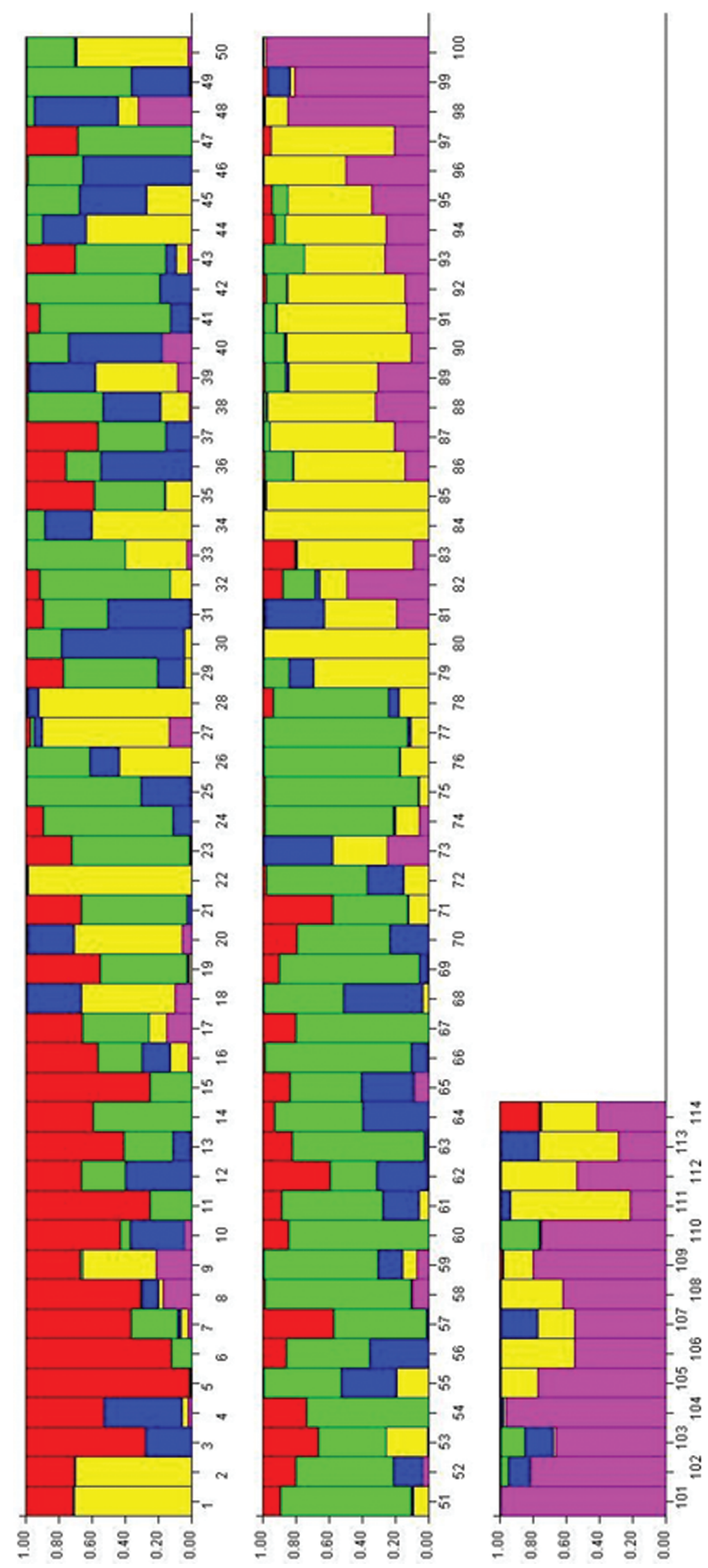

峁 Krishna et al. (2004) with principal compo-

nents analysis two-dimensional plots.

Identification of genetically homogenous groups of individuals using model-based STRUCTURE analysis was attempted. In our study, five discrete subgroups were obtained, with Subgroups 2 (30.7\% of accessions) and 4 ( $25.2 \%$ of accessions) corresponding to the major clusters observed by tree-based on NJ clustering and multivariate PCoA clustering methods. In crop plants, STRUCTURE analysis is routinely employed to identify the presence of subgroups in core collections (Anderson et al., 2009; Abdurakhmonov et al., 2008; Hasan et al., 2008). The five Ks structuring corresponded to the five genetic clusters revealed by PCoA plot and the five distinct clusters detected in NJ dendrograms. Together, NJ, PCoA, and STRUCTURE analysis separated the Valencia accessions into similar groups. However, a tight population STRUCTURE defined by distinct geographic locations could not be found in the Valencia germplasm studied. Furthermore, there were eight accessions from five continents that had admixtures from all five clusters. This could be due to the composition of the Valencia germplasm collected from different parts of the world at different times over the past century. The presence of admixtures in the germplasm is also not surprising as the majority of accessions included are mainly introductions from South America, the center of origin for the Valencia market type.

In summary, the 52 SSR markers detected moderate genetic diversity among the 114 Valencia peanut germplasm accessions. The NJ, PCoA clustering, and STRUCTURE analysis generated distinct groups among the Valencia genotypes. The majority of the accessions originated from Brazil and grouped into a single major cluster, emphasizing the importance of this country as a primary center of origin for the Valencia peanut market type. The molecular diversity observed in the Valencia germplasm provided useful information to aid selection of genetically diverse accessions that could be used for crop improvement. Our future efforts will focus on evaluation of the Valencia germplasm for important agronomic and seed quality traits and for resistance to biotic and abiotic stresses to identify trait-specific, genetically diverse germplasm to broaden the genetic base of Valencia peanut cultivars. 


\section{Acknowledgments}

The authors wish to thank Roy N. Pitman, USDA-ARS Plant Genetic Resources Unit, Griffin, GA, for the germplasm. The authors also thank Halee Hughes, Jacob Sanchez, and Marie Syapin for their technical assistance. This research was supported in part by National Peanut Board, New Mexico Peanut Research Board, New Mexico Agricultural Experiment Station, USDA-ARS CRIS 6208-21000-012-00D, and USAID-Peanut CRSP through University of Georgia ECGA-00-0700001-00.

\section{References}

Abdurakhmonov, I.Y., R.J. Kohel, J.Z. Yu, A.E. Pepper, A.A. Abdullaev, F.N. Kushanov, I.B. Salakhutdinov, Z.T. Buriev, S. Saha, B.E. Scheffler, J.N. Jenkins, and A. Abdukarimov. 2008. Molecular diversity and association mapping of fiber quality traits in exotic G. hirsutum L. germplasm. Genomics 92:478-487.

Anderson, W.F., A. Maas, and P. Ozias-Akins. 2009. Genetic variability of a forage bermudagrass core collection. Crop Sci. 49:1347-1358.

Baichi-Hall, C., R. Keys, and H. Stalker. 1991. Use of protein profiles to characterize peanut cultivars. Newsl. Assoc. Official Seed Anal. 65:25-26.

Barkley, N.D., R. Dean, R.N. Pitman, M.L. Wang, C.C. Holbrook, and G.A. Pederson. 2007. Genetic diversity of cultivated and wild-type peanuts evaluated with M-13 tailed SSR markers and sequencing. Genet. Res. Camb. 89:93-106.

Botstein, D., R.L. White, M. Skolnick, and R.W. Davis. 1980. Construction of a genetic linkage map in man using restriction fragment length polymorphisms. Am. J. Hum. Genet. 32:314-331.

Brown, A.H.D. 1989. Core collections: A practical approach to genetic resource management. Genome 31:818-824.

Cuc, L.M., E.S. Mace, J.H. Crouch, V.D. Quang, T.D. Long, and R.K. Varshney. 2008. Isolation and characterization of novel microsatellite markers and their application for diversity assessment in cultivated groundnut (Arachis hypogaea). BMC Plant Biol. 8:55.

Dwivedi, S.L., N. Puppala, H.D. Upadhyaya, N. Manivannan, and S. Singh. 2008. Developing a core collection of peanut specific to Valencia market type. Crop Sci. 48:625-632.

Falush, D., M. Stephens, and J.K. Pritchard. 2003. Inference of population structure using multilocus genotype data: Linked loci and correlated allele frequencies. Genetics 164:1547-1587.

Falush, D., M. Stephens, and J.K. Pritchard. 2007. Inference of population structure using multilocus genotype data: Dominant markers and null alleles. Mol. Ecol. Notes 7:574-578.

FAOSTAT. 2008. FAOSTAT home page. Available at http://faostat.fao.org/ (verified 25 Jan. 2011). FAO, Rome.

Fávero, A.P., C.E. Simpson, J.F.M. Valls, and N.A. Vello. 2006. Study of the evolution of cultivated peanut through crossability studies among Arachis ipaënsis, A. duranensis, and $A$. hypogaea. Crop Sci. 46:1546-1552.

Ferguson, M.E., P.J. Bramel, and S. Chandra. 2004a. Gene diversity among botanical varieties in peanut (Arachis hypogaea L.). Crop Sci. 44:1847-1854.

Ferguson, M.E., M. Burow, S. Schulze, P. Bramel, A. Paterson, S. Kresovich, and S. Mitchell. 2004b. Microsatellite identification and characterization in peanut ( $A$. hypogaea L.). Theor. Appl. Genet. 108:1064-1070.
Frankel, O.H. 1984. Genetic perspectives of germplasm conservation. p. 161-170. In W.K. Arber et al. (ed.) Genetic manipulation: Impact on man and society. Cambridge Univ. Press, Cambridge, UK.

Freitas, F.O., M.C. Moretzshon, and J.F.M. Valls. 2007. Genetic variability of Brazilian Indian landraces of Arachis hypogaea L. Genet. Mol. Res. 6:675-684.

Gimenes, M.A., A.A. Hoshino, A.V.G. Barbosa, D.A. Palmieri, and C.R. Lopes. 2007. Characterization and transferability of microsatellite markers of the cultivated peanut (Arachis hypogaea). BMC Plant Biol. 7:9.

Gimenes, M.A., C.R. Lopes, M.L. Galgaro, J.F.M. Valls, and G. Kochert. 2002. RFLP analysis of genetic variation in species of section Arachis, genus Arachis (Leguminosae). Euphytica 123:421-429.

Gregory, W.C., A. Krapovickas, and M.P. Gregory. 1980. Structure, variation, evolution and classification in Arachis. p. 469481. In R.J. Summerfield and A.H. Bunting (ed.) Advances in legume science. Royal Botanic Gardens, Kew, UK.

Halward, T., T. Stalker, E. LaRue, and G. Kochert. 1992. Use of single primer DNA amplifications in genetic studies of peanut (Arachis hypogaea L.). Plant Mol. Biol. 18:315-325.

Halward, T.M., H.T. Stalker, E.A. LaRue, and G. Kochert. 1991. Genetic variation detectable with molecular markers among unadapted germplasm resources of cultivated peanut and related wild species. Genome 34:1013-1020.

Hasan, M., W. Friedt, J. Pons-Kühnemann, N.M. Freitag, K. Link, and R.J. Snowdon. 2008. Association of gene-linked SSR markers to seed glucosinolate content in oilseed rape (Brassica napus ssp. napus). Theor. Appl. Genet. 116:1035-1049.

He, G., R. Meng, B. Guo, G. Gao, M. Newman, R.N. Pittman, and C.S. Prakash. 2005. Simple sequence repeat markers for botanical varieties of cultivated peanut (Arachis hypogaea L.). Euphytica 142:131-136.

He, G., R. Meng, M. Newman, G. Gao, R. Pittman, and C.S. Prakash. 2003. Microsatellites as DNA markers in cultivated peanut (A. hypogaea L.). BMC Plant Biol. 3:3.

Herselman, L. 2003. Genetic variation among southern African cultivated peanut (Arachis hypogaea L.) genotypes as revealed by AFLP analysis. Euphytica 133:319-327.

Holbrook, C.C., W.F. Anderson, and R.N. Pitmann. 1993. Selection of core collection from US germplasm collection of peanut. Crop Sci. 33:859-861.

Holbrook, C.C., and W. Dong. 2005. Development and evaluation of a mini core collection for the US peanut germplasm collection. Crop Sci. 45:1540-1544.

Hong, Y., X. Liang, X. Chen, H. Liu, G. Zhou, S. Li, and S. Wen. 2008. Construction of genetic linkage map based on SSR markers in peanut (Arachis hypogaea L.). Agric. Sci. China 78:915-921.

Hopkins, M.S., A.M. Casa, T. Wang, S.E. Mitchell, R. Dean, G.D. Kochert, and S. Kresovich. 1999. Discovery and characterization of polymorphic simple sequence repeats (SSRs) in peanut. Crop Sci. 39:1243-1247.

Hwang, Y.T., Y. Nakamoto, I. Kono, H. Enoki, H. Funatsuki, K. Kitamura, and M. Ishimoto. 2008. Genetic diversity of cultivated and wild soybeans including Japanese elite cultivars as revealed by length polymorphism of SSR markers. Breed. Sci. 58:315-323.

Isleib, T.G., C.C. Holbrook, and D.W. Gorbet. 2001. Use of plant introductions in peanut cultivar development. Peanut Sci. 28:96-113. 
Jiang, H., B. Liao, X. Ren, Y. Lei, E. Mace, T. Fu, and J.H. Crouch. 2007. Comparative assessment of genetic diversity of peanut (Arachis hypogaea L.) with various levels of resistance to bacterial wilt through SSR and AFLP analyses. J. Genet. Genomics 34:544-554.

Khedikar, Y.P., M.V.C. Gowda, C. Sarvamangala, K.V. Patgar, H.D. Upadhyaya, and R.K. Varshney. 2010. A QTL study on late leaf spot and rust revealed one major QTL for molecular breeding for rust resistance in groundnut (Arachis hypogaea L.). Theor. Appl. Genet. 121:971-984.

Knauft, D.A., and D.W. Gorbet. 1989. Genetic diversity among peanut cultivars. Crop Sci. 29:1417-1422.

Kochert, G., T. Halward, W.D. Branch, and C.E. Simpson. 1991. RFLP variability in peanut (Arachis hypogaea L.) cultivars and wild species. Theor. Appl. Genet. 81:565-570.

Kochert, G., H.T. Stalker, M. Ginenes, L. Galgaro, and K. Moore. 1996. RFLP and cytogenetic evidence for the progenitor species of allotetraploid cultivated peanut (Arachis hypogaea L.). Am. J. Bot. 83:1282-1291.

Kottapalli, K.R., M.D. Burow, G. Burow, J. Burke, and N. Puppala. 2007. Molecular characterization of the US peanut mini core collection using microsatellite markers. Crop Sci. 47:1718-1727.

Kottapalli, K.R., R. Rakwal, J. Shibato, G. Burow, D. Tissue, J. Burke, N. Puppala, M. Burow, and P. Payton. 2009. Physiology and proteomics of the water-deficit stress response in three contrasting peanut genotypes. Plant Cell Environ. 32:380-407.

Krapovickas, A., and W.C. Gregory. 1994. Taxonomía del género Arachis (Leguminosae). (In Spanish, with English abstract.) Bonplandia 8:1-186.

Krishna, G.K., J. Zhang, M. Burow, R.N. Pittman, S.G. Delikostadinov, Y. Lu, and N. Puppala. 2004. Genetic diversity analysis in Valencia peanut (Arachis hypogaea L.) using microsatellites. Cell. Mol. Biol. Lett. 9:685-697.

Lacks, G.D., and H.T. Stalker. 1993. Isozyme analysis of Arachis species and interspecific hybrids. Peanut Sci. 20:76-81.

Lanham, P., B. Foster, P. McNicol, J. Moss, and W. Powell. 1994. Seed storage protein variation in Arachis species. Genome 37:487-496.

Litt, M., and J.A. Lutty. 1989. A hypervariable microsatellite revealed by in vitro amplification of a dinucleotide repeats within the cardiac muscle actin gene. Am. J. Hum. Genet. 44:397-401.

Liu, K., and S.V. Muse. 2005. Powermarker: Integrated analysis environment for genetic marker data. Bioinformatics 21:2128-2129.

Mace, E.S., H.K. Buhariwalla, and J.H. Crouch. 2003. A high throughput DNA extraction protocol for tropical molecular breeding programs. Plant Mol. Biol. Rep. 21:459a-459h.

Mace, E.S., D.T. Phong, H.D. Upadhyaya, S. Chandra, and J.H. Crouch. 2006. Simple sequence SSR analysis of cultivated groundnut (Arachis hypgaea L.) germplasm resistant to rust and late leaf spot diseases. Euphytica 152:317-330.

Mace, E.S., W. Yuejin, L. Boshou, H.D. Upadhyaya, S. Chandra, and J.H. Crouch. 2007. Simple sequence repeat (SSR)-based diversity analysis of groundnut (Arachis hypgaea L.) germplasm resistant to bacterial wilt. Plant Genet. Resour. 5:27-36.

Mantel, N.A. 1967. The detection of disease clustering and a generalized regression approach. Cancer Res. 27:209-220.

Milla-Lewis, S.R., M.C. Zuleta, and T.G. Isleib. 2010a. Simple sequence repeat allelic diversity in Virginia-type peanut cultivars released from 1943 to 2006. Crop Sci. 50:1348-1356.

Milla-Lewis, S.R., M.C. Zuleta, and T.G. Isleib. 2010b. Assessment of genetic diversity among US Runner-type peanut cultivars using simple sequence repeat markers. Crop Sci. 50:2396-2405. Moretzsohn, M.C., A.V.G. Barbosa, D.M.T. Alves-Freitas, C.
Teixeira, S.C.M. Leal-Bertioli, C.R. Lopes, M.M. Cavallri, J.F.M. Valls, D.J. Bertioli, and M.A. Gimenes. 2009. A linkage map for the B-genome of Arachis (Fabaceae) and its synteny to the A-genome. BMC Plant Biol. 9:40.

Moretzsohn, M.C., M.S. Hopkins, S.E. Mitchell, S. Kresovich, J.F.M. Valls, and M.E. Ferreira. 2004. Genetic diversity of peanut (Arachis hypogaea L.) and its wild relatives based on the analysis of hypervariable regions of the genome. BMC Plant Biol. 4:11.

Moretzsohn, M.C., L. Leoi, K. Proite, P.M. Guimaraes, S.C.M. Leal-Bertolli, M.A. Gimenes, W.S. Martins, J.F.M. Vall, D. Grattapaglia, and D. Bertiolli. 2005. A microsatellite-based, gene-rich linkage map of the AA genome of Arachis (Fabaceae). Theor. Appl. Genet. 111:1060-1071.

Naito, Y., S. Suzuki, Y. Iwata, and T. Kuboyama. 2008. Genetic diversity and relationship analysis of peanut germplasm using SSR markers. Breed. Sci. 58:293-300.

Nei, M. 1972. Genetic distance between populations. Am. Nat. 106:283-292.

Nei, M. 1973. Analysis of gene diversity in subdivided populations. Proc. Natl. Acad. Sci. USA 70:3321-3323.

Paik-Ro, O.G., R.L. Smith, and D.A. Knauft. 1992. Restriction fragment length polymorphism of six peanut species within the Arachis section. Theor. Appl. Genet. 84:201-208.

Palmieri, D.A., M.D. Bechara, R.A. Curi, and C.R. Lopes. 2005. Novel polymorphic microsatellite markers in section Caulorrhizae (Arachis, Fabaceae). Mol. Ecol. Notes 5:77-79.

Pritchard, J.K., M. Stephens, and P. Donnelly. 2000. Inference of population structure using multilocus genotype data. Genetics 155:945-959.

Proite, K., S.C. Leal-Bertioli, D.J. Bertioli, M.C. Moretzsohn, F.R. da Silva, N.F. Martins, and P.M. Guimaraes. 2007. ESTs from a wild Arachis species for gene discovery and marker development. BMC Plant Biol. 7:7.

Ramamurthy, G.V. 1974. Improved strains of oilseeds in India and their characteristics (statements). Oilseeds J. 4:12-33.

Rohlf, F.J. 2000. NTSYS-PC: Numerical taxonomy and multivariate analysis system. Version 2.2. Exeter Software, Setauket, NY.

Seijo, G., G.I. Lavia, A. Fernández, A. Krapovickas, D.A. Ducasse, D.J. Bertioli, and E.A. Moscone. 2007. Genomic relationships between the cultivated peanut (Arachis hypogaea, Leguminosae) and its close relatives revealed by double GISH. Am. J. Bot. 94:1963-1971.

Tang, R., G. Gao, L. He, Z. Han, S. Shan, R. Zhong, C. Zhou, J. Jiang, Y. Li, and W. Zhuang. 2007. Genetic diversity in cultivated groundnut based on SSR markers. J. Genet. GeN. 34:449-459.

Upadhyaya, H.D., P.J. Bramel, R. Ortiz, and S. Singh. 2002. Developing a mini core of peanut for utilization of genetic resources. Crop Sci. 42:2150-2156.

Upadhyaya, H.D., R. Ortiz, P.J. Bramel, and S. Singh. 2003. Development of a groundnut core collection using taxonomical, geographical and morphological descriptors. Genet. Resour. Crop Evol. 50:139-148.

Upadhyaya, H.D., R.P.S. Pundir, S.L. Dwivedi, and C.L.L. Gowda. 2009. Mini core collections for efficient utilization of plant genetic resources in crop improvement programs. Info. Bull. 78. Int. Crops Res. Inst. for the Semi-Arid Tropics, Patancheru, India.

Varshney, R.K., D.J. Bertioli, M.C. Moretzsohn, V. Vadez, L. Krishnamurthy, R. Aruna, S.N. Nigam, B.J. Moss, K. Seetha, K. Ravi, G. He, S.J. Kanpp, and D.A. Hoisington. 2008. The first SSR-based genetic linkage map for cultivated groundnut (Arachis hypogaea L.). Theor. Appl. Genet. 118:729-739. 